
\title{
3 Research Soure \\ Specification of Interventions and Selection of Control in Acupuncture Randomized Controlled Trials: A Cross-Sectional Study
}

Jiali Liu

Sichuan University West China Hospital

Ling Li

Sichuan University West China Hospital

Xiaochao Luo

Sichuan University West China Hospital

Xuan Qin

Sichuan University West China Hospital

\section{Ling Zhao}

Chengdu University of Traditional Chinese Medicine Wenjiang Campus: Chengdu University of

Traditional Chinese Medicine

Jiping Zhao

Beijing University of Chinese Medicine Affiliated Dongzhimen Hospital

\section{Xu Zhou}

Jiangxi University of Traditional Chinese Medicine

Yanmei Liu

Sichuan University West China Hospital

Ke Deng

Sichuan University West China Hospital

\section{Yu Ma}

Sichuan University West China Hospital

\section{Kang Zou}

Sichuan University West China Hospital

\section{Xin Sun ( $\nabla$ sunxin@wchscu.cn)}

Sichuan University West China Hospital https://orcid.org/0000-0002-6554-7088

\section{Research}

Keywords: Acupuncture, Randomized controlled trials, Specification of interventions, Sham acupuncture, Cross-sectional study. 
Posted Date: November 2nd, 2021

DOI: https://doi.org/10.21203/rs.3.rs-1023037/v1

License: (c) (i) This work is licensed under a Creative Commons Attribution 4.0 International License. Read Full License 


\section{Abstract}

Background: Specification of interventions and selection of control are two methodological determinants for a successful acupuncture trial. However, it is not fully understood about the current practice of these two determinants. We thus conduced a cross-sectional study to examine specification of interventions and selection of control among current acupuncture randomized controlled trials (RCTs).

Methods: We searched PubMed for acupuncture RCTs published in the core clinical journals and complementary and alternative medicine (CAM) journals from January 2010 to December 2019 (10 years), and included RCTs that assessed treatment effects of acupuncture versus any type of control. Teams of methods-trained investigators who had experiences in acupuncture trials independently screened reports for eligibility and collected data, using a prespecified standardized questionnaire. We used network meta-analyses to investigate whether treatment effect was differential in patients with chronic pain when using sham acupuncture as a control versus using waiting-list or no treatment.

Results: Of 319 eligible RCTs, most well specified style of acupuncture (86.8\%), acupoint prescription (96.2\%), type of needle stimulation (90.3\%) and needle retention time (85.6\%). However, other acupuncture details were less specified, including achievement of response sought (65.5\%) and needle manipulation (50.5\%), specification of number of needle insertions (21.9\%), angle and direction of insertion (31.3\%), patients posture (32.3\%) and co-interventions (22.9\%). Sham acupuncture (41.4\%) was the most frequently used control, followed by waiting-list or no treatment (32.9\%). There was no differential treatment effect when using sham acupuncture versus waiting-list/no treatment as a control $(\mathrm{SMD}=-0.15,95 \% \mathrm{Cl}-0.91$ to 0.62$)$.

Conclusions: Over a decade of research practice, important gaps remained in the specification of acupuncture interventions, including specification of response sought, needle manipulation, and cointerventions. While sham acupuncture was largely used, waiting-list or no treatment may also be used as an appropriate control.

\section{Background}

As a complementary and alternative therapy, acupuncture has been widely accepted and used in 103 World Health Organization (WHO) member states [1-5]. Randomized controlled trials (RCTs) are the best evidence about the treatment effects of acupuncture. While an increasing number of acupuncture trials have been published in the past decades [6], their methodological rigors - thus the resulting evidence are often challenged $[7,8]$. Among the nominated concerns, specification of interventions and selection of control are the two major methodological issues that have profound impact on the validity and applicability of acupuncture trials $[9,10]$.

As a complex intervention, the effect of acupuncture is largely affected by design and implementation of interventions, and specification of interventions is a key determinant for a successful acupuncture trial [11-13]. However, these methodological details were not well attended in acupuncture trials. For 
example, a survey published in 2009 found that only $10.5 \%$ of acupuncture trials in top medical journals fully specified acupuncture interventions [14]. Two subsequent surveys also showed incomplete specification of interventions in Korean acupuncture trials [15] and trials for migraine [16]. Up to now, it is not fully understood about how current acupuncture trials specify interventions, and the extent to which the methodological specifications of these trials are met with the established standards (e.g., revised STandards for Reporting Interventions in Clinical Trials of Acupuncture (STRICTA)) [17].

Due to the nature of complex intervention, a valid control in acupuncture trials should minimize placebo effect and nonspecific effect $[18,19]$, and the resulting effect would be attributable to the underlying true effect of acupuncture intervention [20]. In 1997, the NIH proposed to use sham or placebo acupuncture as a control to eliminate the placebo effect [1]. However, controversies continue as to the appropriate choice of control in acupuncture trials. For instance, there has a debate over the choice of appropriate sham acupuncture in trials on pain, as sham acupuncture (such as shallow needling) might have a certain therapeutic effect [20,21].

A comprehensive and systematic survey of trials would enable effective identification of methodological limitations of current acupuncture trials and inform methodological improvement. Therefore, we conducted a cross-sectional study of acupuncture trials published in the last 10 years to investigate how acupuncture trials specify interventions and choose control. We also investigated whether there was a differential effect in trials using sham acupuncture versus waiting-list or no treatment.

\section{Methods}

\section{Eligibility criteria}

We included a study if it was an RCT, assessed the effects of acupuncture (i.e., manual or electrical needle stimulation) versus any type of control treatment, examined efficacy/effectiveness with or without safety outcomes, and was a main study report. We excluded trial protocols, pilot trials, or RCTs reported as a research letter.

\section{Literature search}

We searched PubMed to identify reports of acupuncture RCTs published in the core clinical journals as well as in complementary and alternative medicine (CAM) journals from January 2010 to December 2019 (10 years). The core clinical journals, previously known as the Abridged Index Medicus, were defined by the US National Library of Medicine, and included 118 journals published in English in 2020, covering all specialties of clinical medicine and public health sciences [22]. CAM journals were defined by the SClmago Journal \& Country Rank (SJR) website, and comprised 55 journals indexed in Web of Science in 2019 [23]. We used MeSH and free texts terms related to acupuncture, RCT and journals to search for potentially eligible studies, and one experienced investigator $(\mathrm{JL})$ developed the search strategy with reference to a previous related study [24] (Appendix 1). 


\section{Study process}

Two paired methods-trained researchers, who had experiences in acupuncture trials, used pilot-tested, standardized forms, together with detailed written instructions to screen abstracts and full texts for eligibility, and to abstract data from eligible studies independently and in duplicate. Disagreements were resolved through discussion or, if needed, adjudication by a third researcher (JL or LL).

\section{Data collection}

To assess implementation of interventions and selection of control among acupuncture RCTs, we developed a questionnaire to collect data from included trials. The original version was developed by three experienced authors (JL, LL and XS) with reference to revised STRICTA [17] and previous methodological studies [25-27]. Then our study group piloted the questionnaire, and convened to discuss the appropriateness and applicability of included items. After further revising the questionnaire, we conducted the second pilot and addressed discrepancies through group discussion. Based on the piloted questionnaire, we collected the following information from eligible trials:

1) General characteristics: year of publication, journal type, hypothesis of the trial, type of design, international trial, center involved, country of trial conducted, sample size, length of follow-up, number of authors, methodologists involved, source of funding, study setting, participants recruitment, trial registered, protocol provided, exclusion of patients with acupuncture experience, and Traditional Chinese Medicine (TCM) diagnosis reported.

We judged that a trial involved a methodologist if any author declared an affiliation with a department of epidemiology, statistics, or evidence-based medicine, or if a methodologist was listed in the acknowledgement section.

2) Acupuncture rationale:style of acupuncture specified, style of acupuncture, acupoint prescription, reasoning for acupuncture treatment, rationale for acupoint selection, and standards of acupoint positioning.

3) Acupuncture details: we collected details of needling, treatment regimen, other components of treatment, and practitioner background from eligible trials. Details of needling included needle stimulation, number of needle insertions per subject per session, names of points used, response sought, etc. Treatment regimen included total number of treatment sessions, actual number of treatment sessions specified, incomplete treatment sessions specified, frequency of treatment sessions, duration of treatment sessions, etc. Other components of treatment includeddetails of other interventions administered to the acupuncture group, and setting and context of treatment (i.e., instructions to practitioners, and information and explanations to patients). Practitioner background included description of participating acupuncturists (qualification or professional affiliation, years in acupuncture practice, other relevant experience). 
4) Control selection and rationale.type of control, rationale for the control selection, precise description of the control, and details of sham acupuncture (e.g., sham acupoints, shallow needling, blunt needle device).

\section{5) Main effect of pain outcome in trials of acupuncture for chronic pain: name of pain outcome,} instrument used to measure the pain intensity, mean and standard deviation (SD) of changes form baseline in pain intensity, and number of patients included for analyses in each treatment group. If mean and SD of changes form baseline of pain intensity were not available, we collected mean and SD at baseline and last follow up.

One pain outcome was selected in each trial of acupuncture for chronic pain. If the primary outcome of a trial was pain intensity, we selected it as the primary pain outcome for our analyses; Otherwise, we selected the first reported pain intensity outcome from secondary outcomes for our analyses. If a trial did not clearly specify the primary or secondary outcomes, we selected one outcome according to our previously published strategy $[27,28]$.

\section{Statistical analysis}

We conducted descriptive analyses of study characteristics, rationale and details of acupuncture interventions and control selection of included trials. For all descriptive analyses, we used frequencies (and percentages) for dichotomous variables, and mean (and SD) or median (and range) for continuous variables. We used Chi-Square test or Fisher's exact test to compare characteristics, interventions implementation, and control selection of trials published in core clinical journals and CAM journals for dichotomous variables, and t-test for continuous variables when the distribution was normal or Mann Whitney U test otherwise. We used R software (version 3.6.3) for data analysis, and a P value of 0.05 or less than 0.05 were considered statistically significant.

As most included trial assessing the effects of acupuncture for chronic pain, we conducted network metaanalyses to investigate whether there was a differential effect between sham acupuncture and waitinglist/no treatment groups in patients with chronic pain. We also conducted two separate analyses by type of sham acupuncture (i.e., non-penetrating vs. penetrating) to explore whether non-penetrating or penetrating sham acupuncture had differential effect. The primary outcome was changes form baseline of pain intensity measured by scales such as $10-\mathrm{cm}$ Visual Analog Scale (VAS), and an 11-cm point numeric rating scale (NRS). For trials that did not report changes form baseline in pain intensity, we calculated the mean change from baseline and imputed its SD according to approaches described in Cochrane handbook[29]. We conducted frequentist pairwise, indirect and network meta-analyses using a random-effects model by inverse variance method, with standardized mean difference (SMD) and corresponding $95 \%$ confidence intervals. We used node splitting method to check for consistency of direct and indirect evidence in our network.

\section{Results}


Our search yielded 1850 potentially relevant reports. After screening full text reports 319 RCTs proved eligible, including 51 trials published in core clinical journals and 268 trials in CAM journals (Figure 1).

Table 1 summarized the general characteristics of included trials. Among these 319 trials, the median sample size was 72 (range 10 to 1075), and the median follow-up was 8 weeks (range 0 to 104). Only four were international trials, and $72(22.6 \%)$ were multi-center trials. About half of trials were conducted in China $(n=170,53.3 \%) ; 39(12.2 \%)$ trials explicitly excluded patients with any acupuncture experience. Only 26 (39.5\%) clearly specified trial registration, and 27 (8.5\%) provided study protocols. Compared with trials published in CAM journals, those in core clinical journals included more patients (median $116 \mathrm{vs.}$ $66, P \unrhd 0.001$ ), had a longer follow up (median 16 vs. 8 weeks, $P=0.001$ ). Those trials were also more likely to perform trial registration ( $76.5 \%$ vs. $32.5 \%, \mathrm{P} \bowtie 0.001)$ and provide protocol $(31.4 \%$ vs. $4.1 \%, \mathrm{P} \bowtie 0.001)$.

\subsection{Specification of acupuncture interventions}

Table 2 described the specification of acupuncture rationale. Of these 319 trials, 277 (86.8\%) specified style of acupuncture, among which the most used style was TCM acupuncture (85.9\%); n=294 (92.2\%) provided the reasoning for acupuncture; 307 (96.2\%) provided acupoint prescription, among which $71.3 \%$ used standardized prescriptions and the rest were either partially or fully individualized prescriptions; 215 $(67.4 \%)$ stated the rationale for acupoint selection, among which $80.0 \%$ was based on TCM theory or evolution of patient symptoms. All included trials specified acupoint positioning standards.

Table $3 a$ described the details of needling. Most trials specified the type of needle stimulation $(n=288$, $90.3 \%)$, names of points $(n=297,93.1 \%)$ and needle type (e.g., diameter, length) ( $n=266,83.4 \%)$. Nearly two-thirds of trials $(n=209,65.5 \%)$ stated response sought, among which $82.8 \%$ was de qi sensation. More than half mentioned depth of insertion $(n=195,61.1 \%)$ and needle manipulation $(n=161$, $50.5 \%) .273$ trials (85.6\%) reported needle retention time, with a median of 30 minutes. However, only 70 trials $(21.9 \%)$ specified number of needle insertions, and 100 trials (31.3\%) described angle and direction of insertion.

Table $3 b$ summarized the treatment regimen. Most trials $(n=270,84.6 \%)$ specified the total number, frequency $(n=246,77.1 \%)$ and duration $(n=260,81.5 \%)$ of treatment sessions. However, less than a third of trials mentioned patients posture $(n=103,32.3 \%)$, disinfection $(n=90,28.2 \%)$, and actual number of treatment sessions participants received $(n=42,13.2 \%)$.

Table $3 c$ reported co-interventions and contextual information. Most trials $(n=232,72.7 \%)$ specified setting and context of treatments (i.e., instructions to practitioners, and information and explanations to patients), and more than half of trials ( $n=183,57.4 \%)$ mentioned details of participating acupuncturists. Only 73 trials $(22.9 \%)$ stated the details of co-interventions.

Compared with trials published in CAM journals, more trials published in core clinical journals provided reasoning for acupuncture treatment $(100 \%$ vs. $90.7 \%, P=0.02)$, had a longer duration of treatment sessions (median 8 vs. 4 weeks, $P=0.003)$, and were more likely to specify needle stimulation $(98.0 \%$ vs. 
$88.8 \%, P=0.04)$, needle manipulation (64.7\% vs. $48.1 \%, P=0.03)$, response sought $(82.4 \%$ vs. $62.3 \%$, $\mathrm{P}=0.006)$, and details of participating acupuncturists (76.5\% vs. $53.7 \%, \mathrm{P}=0.003)$. No difference was found in specifying rationale for acupoint selection (78.4\% vs. 65.3\%, $\mathrm{P}=0.067$ ) (Tables 2 and 3 ).

\section{Selection and specification of control}

Among those 319 trials, 222 (69.6\%) included one control group, and 97 (30.4\%) included multiple control groups among which 14 used both sham acupuncture and waiting-list/no treatment controls. In general, sham acupuncture $(41.4 \%)$ was the most frequently used control, followed by waiting-list/no treatment (32.9\%). 142 trials (44.5\%) specified the rationale for the choice of control and justified the choice (Table 4). No difference in the specification of control was found between core clinical journals versus CMA journals.

Among the 132 trials with sham acupuncture control, 130 (98.5\%) described the specific type of sham acupuncture, of which 74 (56.9\%) were invasive (penetrating the skin) and 56 (43.1\%) were non-invasive (non-penetrating). Sham points $(n=70,53.8 \%)$ were the most frequently used type of sham acupuncture, followed by non-penetrating sham needle $(n=44,33.8 \%)$ and shallow needling $(n=43,33.1 \%)$ (Figure 2$)$. Details about sham manual and electrical acupuncture were shown in Appendix 2 and Appendix 3.

\section{Choice of sham acupuncture versus waiting-list/no treatment as a control}

We included 28 randomized controlled trials that used either sham acupuncture or waiting-list/no treatment as a control to assess the effects of acupuncture for chronic pain, involving a total of 2,431 patients. Network meta-analysis showed no differential effect between sham acupuncture and waitinglist/no treatment groups in patients with chronic pain (SMD $=-0.15,95 \% \mathrm{Cl}-0.91$ to 0.62 ), while there were statistical differences between acupuncture vs. sham acupuncture ( $S M D=1.46,95 \% \mathrm{Cl} 0.92$ to 2.06), as well as acupuncture vs. waiting-list/no treatment ( $S M D=1.34,95 \% \mathrm{Cl} 0.80$ to 1.89) (Figure 3). No differential effects were found in non-penetrating sham acupuncture (SMD $=0.2,95 \% \mathrm{Cl}-064$ to 1.04$)$ and penetrating sham acupuncture (SMD $=-0.60,95 \% \mathrm{Cl} 1.64$ to 0.43$)$ compared to waiting-list/no treatment (Appendix 4 and Appendix 5).

\section{Discussion}

\section{Main findings and interpretations}

In this systematic survey of acupuncture trials, we found that most trials clearly specified style of acupuncture, acupoint prescription, type of needle stimulation, needle retention time, frequency and duration of treatment sessions. However, other acupuncture details were less specified, including achievement of response sought and needle manipulation, specification of number of needle insertions, angle and direction of insertion, and co-interventions, which should be given more emphasis. We also found that, only $24.8 \%$ trials involved methodologists, and $39.5 \%$ clearly specified trial registration, which also highlighted important areas for improved planning and conduct of acupuncture trials. 
Most included acupuncture trials used TCM acupuncture, and nearly half of included trials were conducted in foreign countries, which indicated that acupuncture is gradually accepted in other countries recently. We also found that $71.3 \%$ acupuncture trials used standardized acupoint prescription, and only $18.2 \%$ used partially individualized acupoint prescription (i.e., fixed acupoint combined with acupoint selected by syndrome differentiation), which might not fully reflect the feature of TCM interventions (i.e., treatment based on syndrome differentiation), and thus other epidemiological design such as pragmatic randomized controlled trials can be used to increase consideration of TCM features [30,31].

Moreover, we found that trials published in core clinical journals were superior to trials published in CAM journals in terms of certain acupuncture details (such as needle manipulation, response sought, and participating acupuncturists), suggesting that editors in CAM journals should consider requiring acupuncture trials authors to adhere to the STRICTA checklist regarding the reporting of acupuncture interventions.

In addition, our findings showed no differential effect between sham acupuncture and waiting-list/no treatment for chronic pain, which was consistent with findings from one previous study, including 202 placebo controlled trials with 16566 patients and showing no clinical effects of placebo

interventions [32]. However, criticism of acupuncture sham control has existed for decades because sham acupuncture is not physiologically inert [33]. There were several kinds of sham acupuncture procedures, thus the effects of different sham acupuncture (e.g., non-penetrating vs. penetrating sham acupuncture) may differ [34]. Unfortunately, our study did not find significant differences due to limited number of included studies and lack of direct evidence. While a recent network meta-analysis [35], including eight trials, showed that shallow needling (one kind of penetrating sham acupuncture) was more effective than non-penetrating sham needle (SMD $-7.27,95 \% \mathrm{Cl}-9.11$ to -5.43 ). Therefore, future studies identifying more studies will be necessary to definitively establish or refute possible differences in the effect of sham acupuncture versus waiting-list/no treatment, as well as differences in the effect of different sham acupuncture.

\section{Suggestions for conducting acupuncture trials}

As acupuncture is a complex intervention, acupuncture trials require more involvement of methodologists. Acupuncture trials authors should consider to adhere to the STRICTA checklist to carefully plan and report acupuncture rationale and details, such as response sought, needle manipulation, angle and direction of insertion, and co-interventions.

As we did not find differential effect between sham acupuncture and waiting-list/no treatment for chronic pain, and a previous RCT also showed that a non-penetrating sham needle could successfully blind participants and might be a credible sham control [36], acupuncture trialists should chose an appropriate control according to aims of a study, e.g, a waiting-list/no treatment control selected for aim of controlling the effect of disease natural progression (i.e, natural history, regression to mean), and a nonpenetrating sham acupuncture (i.e., non-penetrating sham needle) control selected for aim of eliminating 
the non-specific effects of needling, which is particularly for studying of pain-related symptoms/disorders [2].

\section{Strengths and limitations}

Our study has several strengths. We used rigorous methods for searching, selecting, and collecting data from eligible trials. Further, our survey included 319 trials from 2010 to 2019 (10 yeas) with a large sample size and relatively good representative. Thirdly, we examined methodological safeguards of acupuncture interventions and control selection reported by acupuncture trials published in core clinical journals and CAM journals systematically.

Our study also has limitations. Firstly, our survey is based on reporting. Authors of acupuncture trials might have pursued rigorous approaches for specification of interventions and selection of control, but they are not explicitly reported in the full text, which may influence our judgment. Secondly, we included a relatively small number of trials to assess the effect sham acupuncture, and although we used standardized mean differences to combine pain intensity data from different chronic pain diseases, heterogeneity between studies may remain due to different chronic pain mechanisms and clinical settings, which may compromise findings from our study.

\section{Conclusions}

Over a decade of research practice, important gaps remained in the specification of acupuncture interventions, including achievement of response sought and needle manipulation, specification of number of needle insertions, angle and direction of insertion and co-interventions. We did not find differential effect between sham acupuncture and waiting-list/no treatment for chronic pain. While sham acupuncture was largely used, waiting list or no treatment may also be used as an appropriate control.

\section{Declarations}

\section{Acknowledgments}

We thank all of the colleagues who were attending in this study and the research assistants, acupuncturists, and supporters of this study.

\section{Authors' contributions}

XS conceived the study. XS acquired the funding. JL, LL and XS had full access to all of the data in the study, and take responsibility for the integrity of the data and the accuracy of the data analysis. JL, ZL, $K D, Y L$, and $Y M$ conducted the literature searches and extracted the data. $J L, L L$ and $X S$ conducted the analysis and interpreted the data. $L L$, JL and XS drafted the manuscript. XS, LL, JWB, BM, XZ, XL, and KZ critically revised the manuscript. All authors read and approved the final manuscript.

\section{Funding}


This study was supported by National Key R\&D Program of China (Grant No. 2017YFC1700406), National Natural Science Foundation of China (Grant No. 81590955), Sichuan Youth Science and Technology Innovation Research Team (Grant No. 2020JDTD0015), China Center for Evidence Based Traditional Chinese Medicine (Grant No. 2020YJSZX-3), and 1.3.5 project for disciplines of excellence, West China Hospital, Sichuan University (Grant No. ZYYC08003). None of the funders had any role in the design and conduct of the study; collection, analysis, and interpretation of the data; preparation, review, or approval of the manuscript; and decision to submit the manuscript for publication.

\section{Competing interests}

The authors declare that they have no competing interests.

\section{Ethics approval and consent to participate}

Not applicable

\section{Consent for publication}

Not applicable

\section{Availability of data and materials}

All data generated or analysed during this study are included in this published article and its supplementary information files.

\section{References}

1. NIH. NIH Consensus Conference. Acupuncture. JAMA. 1998;280(17):1518-24.

2. Chen $H$, Yang M, Ning Z, Lam WL, Zhao YK, Yeung WF, et al. A Guideline for Randomized Controlled Trials of Acupuncture. The American journal of Chinese medicine. 2019;47(1):1-18.

3. Du Y, Li j, Sun D, Li G, Lin X, Ren H, et al. Study on modern disease menu of acupuncture and moxibustion therapy in China. Chinese Acupunture \& Moxibustion. 2007;27(5):373-8.

4. WHO. WHO traditional medicine strategy: 2014-2023.

https://www.who.int/medicines/publications/traditional/trm_strategy14_23/en/. 2014.

5. Scognamillo-Szabó M, Bechara G. Acupuncture: history, basic principles and its use in Veterinary Medicine. Ciência Rural. 2010;40:461-70.

6. Ma Y, Dong M, Zhou K, Mita C, Liu J, Wayne PM. Publication trends in acupuncture research: a 20year bibliometric analysis based on PubMed. PloS one. 2016;11(12):e0168123.

7. Shuai P, Zhou XH, Lao L, Li X. Issues of design and statistical analysis in controlled clinical acupuncture trials: an analysis of English-language reports from Western journals. Statistics in medicine. 2012;31(7):606-18. 
8. Elorriaga Claraco A, Hanna SE, Fargas-Babjak A. Reporting of clinical details in randomized controlled trials of acupuncture for the treatment of migraine/headaches and nausea/vomiting. Journal of alternative and complementary medicine (New York, NY). 2003;9(1):151-9.

9. White AR, Filshie J, Cummings TM. Clinical trials of acupuncture: consensus recommendations for optimal treatment, sham controls and blinding. Complementary therapies in medicine. 2001;9(4):237-45.

10. MacPherson H, White A, Cummings M, Jobst K, Rose K, Niemtzow R. Standards for reporting interventions in controlled trials of acupuncture: the STRICTA recommendations. Complementary therapies in medicine. 2001;9(4):246-9.

11. Yu Z, Luo L, Li Y, Wu Q, Deng S, Lian S, et al. Different manual manipulations and electrical parameters exert different therapeutic effects of acupuncture. Journal of traditional Chinese medicine $=$ Chung $\mathrm{i}$ tsa chih ying wen pan. 2014;34(6):754-8.

12. Vickers AJ, Vertosick EA, Lewith G, MacPherson H, Foster NE, Sherman KJ, et al. Do the effects of acupuncture vary between acupuncturists? Analysis of the Acupuncture Trialists' Collaboration individual patient data meta-analysis. Acupuncture in medicine : journal of the British Medical Acupuncture Society. 2021;39(4):309-17.

13. MacPherson H, Maschino AC, Lewith G, Foster NE, Witt CM, Vickers AJ. Characteristics of acupuncture treatment associated with outcome: an individual patient meta-analysis of 17,922 patients with chronic pain in randomised controlled trials. PloS one. 2013;8(10):e77438.

14. Zheng $H$, Liang F, Li Y. Features of acupuncture randomised controlled trials published in the top four

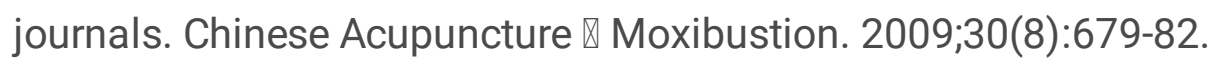

15. Kim KH, Kang JW, Lee MS, Lee JD. Assessment of the quality of reporting in randomised controlled trials of acupuncture in the Korean literature using the CONSORT statement and STRICTA guidelines. BMJ open. 2014;4(7):e005068.

16. Lu T, Lu C, Li H, Xing X, Deng X, Li X, et al. The reporting quality and risk of bias of randomized controlled trials of acupuncture for migraine: methodological study based on STRICTA and RoB 2.0. Complementary therapies in medicine. 2020;52:102433.

17. MacPherson H, Altman DG, Hammerschlag R, Youping L, Taixiang W, White A, et al. Revised STandards for Reporting Interventions in Clinical Trials of Acupuncture (STRICTA): extending the CONSORT statement. PLoS medicine. 2010;7(6):e1000261.

18. Langevin HM, Hammerschlag R, Lao L, Napadow V, Schnyer RN, Sherman KJ. Controversies in acupuncture research: selection of controls and outcome measures in acupuncture clinical trials. Journal of alternative and complementary medicine (New York, NY). 2006;12(10):943-53.

19. Chen H, Ning Z, Lam WL, Lam W-Y, Zhao YK, Yeung JWF, et al. Types of Control in Acupuncture Clinical Trials Might Affect the Conclusion of the Trials: A Review of Acupuncture on Pain Management. Journal of Acupuncture and Meridian Studies. 2016;9(5):227-33.

20. Leon AC, Davis LL, Kraemer HC. The role and interpretation of pilot studies in clinical research. Journal of psychiatric research. 2011;45(5):626-9. 
21. Birch S. A review and analysis of placebo treatments, placebo effects, and placebo controls in trials of medical procedures when sham is not inert. Journal of alternative and complementary medicine (New York, NY). 2006;12(3):303-10.

22. NLM. U.S. National Library of Medicine. Abridged Index Medicus (AIM or "Core Clinical") Journal Titles. http://www.nlm.nih.gov/bsd/aim.html. 2020.

23. SJR. SCImago Journal and Country Rank. Journal ranks. https://www.scimagojr.com/. 2020.

24. Sun X, Briel M, Busse JW, Akl EA, You JJ, Mejza F, et al. Subgroup Analysis of Trials Is Rarely Easy (SATIRE): a study protocol for a systematic review to characterize the analysis, reporting, and claim of subgroup effects in randomized trials. Trials. 2009;10:101.

25. Sun X, Briel M, Busse JW, You JJ, Akl EA, Mejza F, et al. The influence of study characteristics on reporting of subgroup analyses in randomised controlled trials: systematic review. Bmj. 2011;342:d1569.

26. Akl EA, Briel M, You JJ, Sun X, Johnston BC, Busse JW, et al. Potential impact on estimated treatment effects of information lost to follow-up in randomised controlled trials (LOST-IT): systematic review. Bmj. 2012;344:e2809.

27. Li L, Deng K, Busse JW, Zhou X, Xu C, Liu Z, et al. A systematic survey showed important limitations in the methods for assessing drug safety among systematic reviews. Journal of clinical epidemiology. 2020;123:80-90.

28. Bala MM, Akl EA, Sun X, Bassler D, Mertz D, Mejza F, et al. Randomized trials published in higher vs. lower impact journals differ in design, conduct, and analysis. Journal of clinical epidemiology. 2013;66(3):286-95.

29. Higgins JPT, Thomas J, Chandler J, Cumpston M, Li T, Page MJ, et al. Cochrane Handbook for Systematic Reviews of Interventions version 6.2 (updated February 2021). 2021.

30. Loudon K, Treweek S, Sullivan F, Donnan P, Thorpe KE, Zwarenstein M. The PRECIS-2 tool: designing trials that are fit for purpose. Bmj. 2015;350:h2147.

31. Schwartz D, Lellouch J. Explanatory and pragmatic attitudes in therapeutical trials. Journal of clinical epidemiology. 2009;62(5):499-505.

32. Hróbjartsson A, Gøtzsche PC. Placebo interventions for all clinical conditions. The Cochrane database of systematic reviews. 2010;2010(1):Cd003974.

33. Lundeberg T, Lund I, Näslund J, Thomas M. The Emperors sham - wrong assumption that sham needling is sham. Acupuncture in medicine : journal of the British Medical Acupuncture Society. 2008;26(4):239-42.

34. Jiang $Y$, Yin L, Wang Y, Shan C, Liu Y, Xu Y, et al. Assessments of different kinds of sham acupuncture applied in randomized controlled trials. Journal of Acupuncture and Tuina Science. 2011;9(4):199.

35. Kim T-H, Lee MS, Alraek T, Birch S. Acupuncture in sham device controlled trials may not be as effective as acupuncture in the real world: a preliminary network meta-analysis of studies of 
acupuncture for hot flashes in menopausal women. Acupuncture in medicine : journal of the British Medical Acupuncture Society. 2020;38(1):37-44.

36. Lee H, Bang H, Kim Y, Park J, Lee S, Lee H, et al. Non-penetrating sham needle, is it an adequate sham control in acupuncture research? Complementary therapies in medicine. 2011;19:S41-S8.

\section{Tables}

Table 1 General characteristics of included trials 
Characteristics

Total Core clinical

CAM journals $(n=319, \%)$ journals $(n=51, \quad(n=268, \%)$

\%)

value

Study hypothesis

$\square$

$0.001^{*}$

Equivalence

Superiority

Non-inferiority

Not specified

Type of design

Parallel

Cross-over

Factorial

International trial

Center involved

Single center

Multicenter

Not specified

China

Other countries

Sample size

Length of follow-up ${ }^{\#}$

$\leq 8$ weeks

\section{Country of trial conducted \\ China}

China

\begin{tabular}{|c|}
\hline Sample size $\mathrm{e}^{\#}$ \\
\hline Length of follow-up \\
\hline
\end{tabular}

5 (1.5) $\quad 3(5.9)$

$2(0.7)$

52

(16.3)

$4(1.3)$

17 (33.3)

35 (13.1)

258

(80.9)

$\begin{array}{ll}2(3.9) & 2(0.7) \\ 29(56.9) & 229(85.4)\end{array}$

(80.9)

$4(1.3)$
258
$(80.9)$

308

(96.5)

50 (98)

8 (2.5) $\quad 0(0)$

8 (3.0)

$3(0.9) \quad 1(2.0)$

2 (0.7)

4 (1.3) $\quad 2$ (3.9)

2 (0.7)

$0.122^{*}$

$0.264^{*}$

258 (96.3)
198

(62.1)

72

(22.6)

$\begin{array}{ll}49 & 1(2.0) \\ (15.4) & \end{array}$
$26(51.0)$

$24(47.0)$
$172(64.2)$

48 (17.9)

48 (17.9)

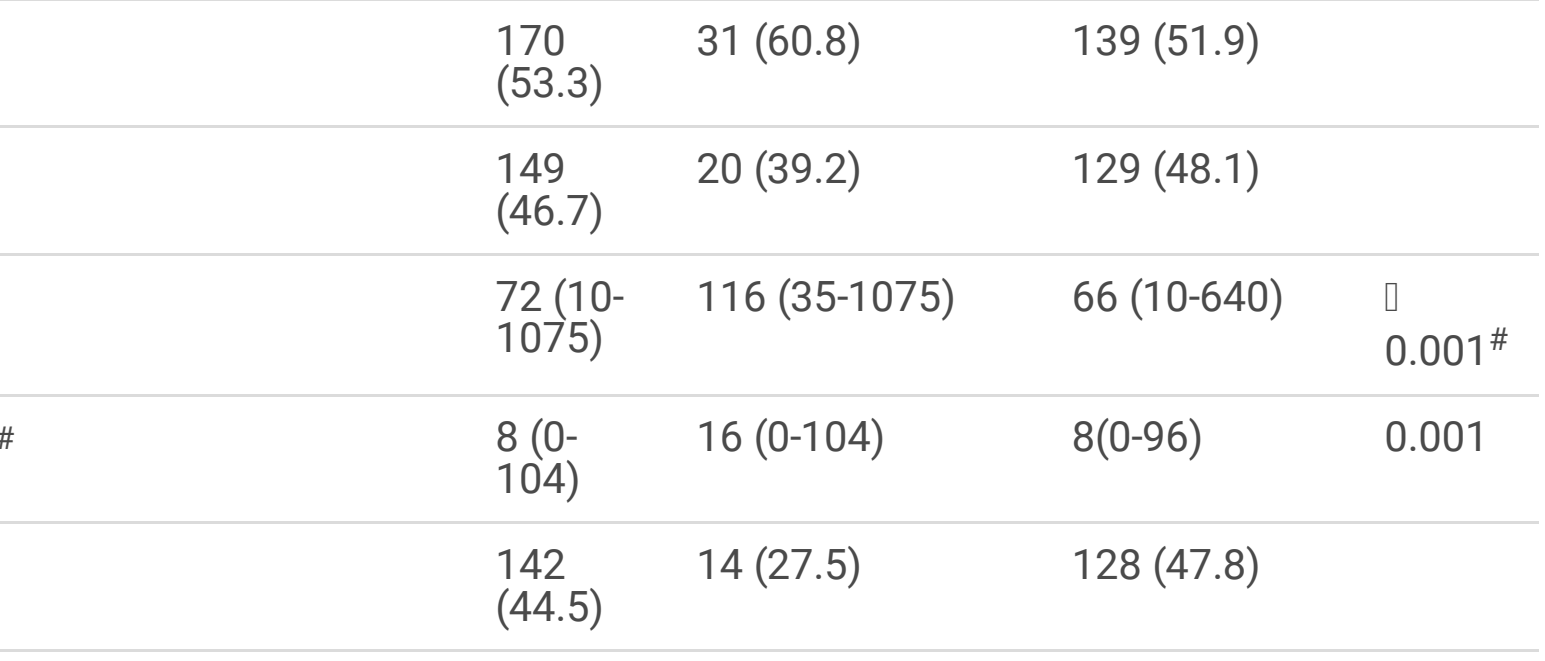




\begin{tabular}{|c|c|c|c|c|}
\hline$>8$ weeks & $\begin{array}{l}129 \\
(40.4)\end{array}$ & $33(64.7)$ & $96(35.8)$ & \\
\hline Not specified & $\begin{array}{l}48 \\
(15.1)\end{array}$ & $4(7.8)$ & $44(16.4)$ & \\
\hline Number of authors" & $\begin{array}{l}6(1- \\
35)\end{array}$ & $8(3-35)$ & $6(1-23)$ & $\begin{array}{l}0.001^{\#} \\
\text {. }\end{array}$ \\
\hline Methodologists involved & $\begin{array}{l}79 \\
(24.8)\end{array}$ & $21(41.2)$ & $58(21.6)$ & 0.003 \\
\hline Source of funding & & & & $0.002^{*}$ \\
\hline Non-profit funding & $\begin{array}{l}204 \\
(63.9)\end{array}$ & $42(82.4)$ & $162(60.4)$ & \\
\hline For profit funding & $4(1.3)$ & $0(0)$ & $4(1.5)$ & \\
\hline No funding & $\begin{array}{l}16 \\
(5.0)\end{array}$ & $4(7.8)$ & $12(4.5)$ & \\
\hline Not reported & $\begin{array}{l}95 \\
(29.7)\end{array}$ & $5(9.8)$ & $90(33.6)$ & \\
\hline Study setting & & & & $0.002^{*}$ \\
\hline Hospital & $\begin{array}{l}229 \\
(71.8)\end{array}$ & $45(88.2)$ & $184(68.7)$ & \\
\hline Community/primary care/private clinic & $\begin{array}{l}32 \\
(10.0)\end{array}$ & $4(7.9)$ & $28(10.4)$ & \\
\hline Others & $\begin{array}{l}15 \\
(4.7)\end{array}$ & $2(3.9)$ & $13(4.9)$ & \\
\hline Not specified & $\begin{array}{l}43 \\
(13.5)\end{array}$ & 0 & $43(16.0)$ & \\
\hline Patient recruitment ${ }^{\&}(n=88)$ & & & & $0.751^{*}$ \\
\hline Poster/brochures & $\begin{array}{l}36 \\
(40.9)\end{array}$ & $10(47.6)$ & $26(38.8)$ & \\
\hline Social media & $\begin{array}{l}43 \\
(48.9)\end{array}$ & $14(66.7)$ & $29(43.3)$ & \\
\hline $\begin{array}{l}\text { Text messaging/ } \\
\text { mailing/telephone }\end{array}$ & $\begin{array}{l}14 \\
(15.9)\end{array}$ & $4(19)$ & $10(14.9)$ & \\
\hline Presentation by researcher & $7(8)$ & $2(9.5)$ & $5(7.5)$ & \\
\hline Doctor recommended & $\begin{array}{l}31 \\
(35.2)\end{array}$ & $6(28.6)$ & $25(37.3)$ & \\
\hline Other & $4(4.5)$ & $0(0)$ & $4(6)$ & \\
\hline
\end{tabular}




\section{Trial registered}

Protocol provided

27

(8.5)

$16(31.4)$

$11(4.1)$

$\square$

$0.001^{*}$

\section{Excluded patients with acupuncture} experience

Yes, excluded patients with any acupuncture experience

Yes, excluded patients with acupuncture experience in a defined duration

No/not specified

TCM diagnosis reported

$$
39
$$

45

(14.1)

235

(73.7)

\section{9}

(9.1)
$10(19.6) \quad 29(10.8)$

$14(27.5) \quad 31(11.6)$

$27(52.9) \quad 208(77.6)$

$2(3.9)$

$27(10.1)$

$0.194^{\star}$

*Fisher's exact test; others are Pearson's chi-squared test. \# Values are median (range) and P value are from Mann-Whitney U test. \& Twenty-eight trials used two approaches, eight trials used three approaches and one trial used four approaches to recruit participants.

Table 2 Acupuncture rationale of included trials 


\begin{tabular}{|c|c|c|c|c|}
\hline Characteristics & $\begin{array}{l}\text { Total } \\
(n=319, \\
\%)\end{array}$ & $\begin{array}{l}\text { Core clinical } \\
\text { journals } \\
(n=51, \%)\end{array}$ & $\begin{array}{l}\text { CAM journals } \\
(n=268, \%)\end{array}$ & $\begin{array}{l}P \\
\text { value }\end{array}$ \\
\hline Style of acupuncture specified & $\begin{array}{l}277 \\
(86.8)\end{array}$ & $48(94.1)$ & $229(85.4)$ & 0.093 \\
\hline \multicolumn{5}{|l|}{ Style of acupuncture $(n=277)^{\#}$} \\
\hline TCM acupuncture & $\begin{array}{l}238 \\
(85.9)\end{array}$ & $38(79.2)$ & $200(87.3)$ & \\
\hline Japanese acupuncture & $2(0.7)$ & $0(0)$ & $2(0.9)$ & \\
\hline Korean acupuncture & $8(2.9)$ & $0(0)$ & $8(3.5)$ & \\
\hline Western medicine acupuncture & $\begin{array}{l}32 \\
(11.6)\end{array}$ & $22(45.8)$ & $10(4.4)$ & \\
\hline $\begin{array}{l}\text { Reasoning for acupuncture } \\
\text { provided }\end{array}$ & $\begin{array}{l}294 \\
(92.2)\end{array}$ & $51(100.0)$ & $243(90.7)$ & $0.02^{*}$ \\
\hline \multicolumn{5}{|l|}{$\begin{array}{l}\text { Reasoning for acupuncture } \\
(n=294)^{\&}\end{array}$} \\
\hline Historical context & $\begin{array}{l}33 \\
(11.2)\end{array}$ & $6(11.8)$ & $27(11.1)$ & \\
\hline Literature sources & $\begin{array}{l}282 \\
(95.9)\end{array}$ & $47(92.2)$ & $235(96.7)$ & \\
\hline Consensus methods & $22(7.5)$ & $11(21.6)$ & $11(4.5)$ & \\
\hline Other & $11(3.7)$ & $4(7.8)$ & $7(2.9)$ & \\
\hline Acupoint prescription provided & $\begin{array}{l}307 \\
(96.2)\end{array}$ & $50(98)$ & $257(95.9)$ & $0.699^{\star}$ \\
\hline \multicolumn{5}{|l|}{ Acupoint prescription $(n=307)$} \\
\hline Standardized acupoint prescription & $\begin{array}{l}219 \\
(71.3)\end{array}$ & $32(64)$ & $187(72.8)$ & \\
\hline Partially individualized prescription & $\begin{array}{l}56 \\
(18.2)\end{array}$ & $8(16)$ & $48(18.7)$ & \\
\hline Fully individualized prescription & $\begin{array}{l}32 \\
(10.5)\end{array}$ & $10(20)$ & $22(8.7)$ & \\
\hline $\begin{array}{l}\text { Rationale for acupoint } \\
\text { selection provided }\end{array}$ & $\begin{array}{l}215 \\
(67.4)\end{array}$ & $40(78.4)$ & $175(65.3)$ & 0.067 \\
\hline \multicolumn{5}{|l|}{$\begin{array}{l}\text { Rationale for acupoint } \\
\text { selection }(n=215)^{\$}\end{array}$} \\
\hline Literature reports/ meta-analysis & 53 & $10(25)$ & $43(24.6)$ & \\
\hline
\end{tabular}


Clinical experience/expert consensus

TCM theory/evolution of patient symptoms

Modern medicine theory

Standards of acupoint positioning

Chinese standard (i.e. GB 1234690)

WHO standard acupuncture locations

Myofascial trigger points

*Fisher's exact test; others are Pearson's chi-squared test. ${ }^{\#}$ Three trials used two styles of
acupuncture. ${ }^{\&}$ Forty-four trials used two reasons for acupuncture treatment, and five trials
reasons for acupuncture treatment. ${ }^{\$}$ Forty-four trials specified two rationales for acupoint
five trials specified three rationales for acupoint selection. acupuncture. \&Forty-four trials used two reasons for acupuncture treatment, and five trials used three

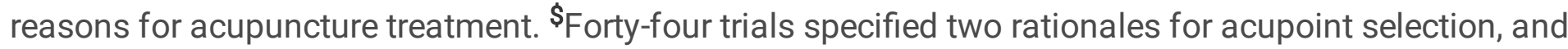

*Fisher's exact test; others are Pearson's chi-squared test.
acupuncture. ${ }^{\&}$ Forty-four trials used two reasons for acupund
reasons for acupuncture treatment. ${ }^{\$}$ Forty-four trials specifie
five trials specified three rationales for acupoint selection.

Table 3 Acupuncture details of included trials
$16(9.1)$

$141(80.6)$

$11(6.3)$

\section{$0.028^{*}$}

275
$(86.2)$

$12(3.8)$

$32(10)$

$10(19.6)$

$3(5.9)$
$237(88.4)$

$9(3.4)$

$22(8.2)$

$38(74.5) \quad 237(88.4)$

$(3.4)$

$22(8.2)$

\section{Table 3 Acupuncture details of included trials}




\begin{tabular}{|c|c|c|c|c|}
\hline Characteristics & $\begin{array}{l}\text { Total } \\
(n=319, \\
\%)\end{array}$ & $\begin{array}{l}\text { Core clinical } \\
\text { journals } \\
(n=51, \%)\end{array}$ & $\begin{array}{l}\text { CAM journals } \\
(n=268, \%)\end{array}$ & $\begin{array}{l}P \\
\text { value }\end{array}$ \\
\hline \multicolumn{5}{|l|}{ Table 3a: Details of needling } \\
\hline Needle stimulation specified & $\begin{array}{l}288 \\
(90.3)\end{array}$ & $50(98.0)$ & $238(88.8)$ & $0.04^{*}$ \\
\hline Needle stimulation $(n=288)$ & & & & 0.23 \\
\hline Manual needle stimulation & $\begin{array}{l}174 \\
(60.4)\end{array}$ & $30(60)$ & $144(60.5)$ & \\
\hline Electrical needle stimulation & $\begin{array}{l}48 \\
(16.7)\end{array}$ & $5(10)$ & $43(18.1)$ & \\
\hline $\begin{array}{l}\text { Both Manual and electrical needle } \\
\text { stimulation }\end{array}$ & $\begin{array}{l}66 \\
(22.9)\end{array}$ & $15(30)$ & $51(21.4)$ & \\
\hline Number of needle insertions specified & $\begin{array}{l}70 \\
(21.9)\end{array}$ & $12(23.5)$ & $58(21.6)$ & 0.765 \\
\hline Names of points used provided & $\begin{array}{l}297 \\
(93.1)\end{array}$ & $47(92.2)$ & $250(93.3)$ & $0.764^{*}$ \\
\hline Needle unilateral/bilateral mentioned & $\begin{array}{l}177 \\
(55.5)\end{array}$ & $31(60.8)$ & $146(54.5)$ & 0.406 \\
\hline Depth of insertion specified & $\begin{array}{l}195 \\
(61.1)\end{array}$ & $33(64.7)$ & $162(60.4)$ & 0.567 \\
\hline $\begin{array}{l}\text { Angle and direction of } \\
\text { insertion described }\end{array}$ & $\begin{array}{l}100 \\
(31.3)\end{array}$ & $15(29.4)$ & $85(31.7)$ & 0.745 \\
\hline Response sought stated & $\begin{array}{l}209 \\
(65.5)\end{array}$ & $42(82.4)$ & $167(62.3)$ & 0.006 \\
\hline Response sought $(n=209)$ & & & & $0.044^{*}$ \\
\hline de qi sensation & $\begin{array}{l}173 \\
(82.8)\end{array}$ & $32(76.2)$ & $140(83.8)$ & \\
\hline Muscle twitch/contraction response & $15(7.2)$ & $7(16.7)$ & $8(4.8)$ & \\
\hline Other & $21(10)$ & $3(7.1)$ & $18(10.8)$ & \\
\hline Needle manipulation specified & $\begin{array}{l}161 \\
(50.5)\end{array}$ & $33(64.7)$ & $129(48.1)$ & 0.03 \\
\hline Needle manipulation $(n=161)^{\&}$ & & & & $0.007 *$ \\
\hline Lifting and thrusting & $\begin{array}{l}55 \\
(34.2)\end{array}$ & $9(27.3)$ & $46(35.7)$ & \\
\hline Twisting and rotating & $\begin{array}{l}104 \\
(64.6)\end{array}$ & $16(48.5)$ & $88(68.2)$ & \\
\hline
\end{tabular}




\begin{tabular}{|c|c|c|c|c|}
\hline Reinforcing and reducing & $\begin{array}{l}24 \\
(14.9)\end{array}$ & $2(6.1)$ & $22(17.1)$ & \\
\hline Other & $\begin{array}{l}34 \\
(21.1)\end{array}$ & $14(42.4)$ & $20(15.5)$ & \\
\hline Needle retention time specified & $\begin{array}{l}273 \\
(85.6)\end{array}$ & $43(84.3)$ & $230(85.8)$ & 0.779 \\
\hline Needle retention time $(\mathrm{min})^{\#}$ & $\begin{array}{l}30(5- \\
90)\end{array}$ & $30(5-90)$ & $30(5-60)$ & $0.274^{\#}$ \\
\hline \15min & $9(3.3)$ & $4(9.3)$ & $5(2.2)$ & \\
\hline 15-30min & $\begin{array}{l}245 \\
(89.7)\end{array}$ & $37(86)$ & $208(90.4)$ & \\
\hline$\otimes 30 \min$ & $19(7)$ & $2(4.7)$ & $17(7.4)$ & \\
\hline $\begin{array}{l}\text { Needle type (e.g., diameter, length) } \\
\text { specified }\end{array}$ & $\begin{array}{l}266 \\
(83.4)\end{array}$ & $44(86.3)$ & $222(82.8)$ & 0.545 \\
\hline $\begin{array}{l}\text { Details of electrical stimulation } \\
(n=114)^{\$}\end{array}$ & & & & $0.664^{*}$ \\
\hline Electrical equipment & $\begin{array}{l}91 \\
(79.8)\end{array}$ & $15(75)$ & $76(80.9)$ & \\
\hline Electric current & $\begin{array}{l}52 \\
(45.6)\end{array}$ & $12(60)$ & $40(42.6)$ & \\
\hline Frequency settings & $\begin{array}{l}95 \\
(83.3)\end{array}$ & $19(95)$ & $76(80.9)$ & \\
\hline Other & $4(3.5)$ & $1(5)$ & $3(3.2)$ & \\
\hline \multicolumn{5}{|l|}{ Table 3b: Treatment regimen } \\
\hline $\begin{array}{l}\text { Total number of treatment sessions } \\
\text { specified }\end{array}$ & $\begin{array}{l}270 \\
(84.6)\end{array}$ & $44(86.3)$ & $226(84.3)$ & 0.724 \\
\hline $\begin{array}{l}\text { Total number of treatment } \\
\text { sessions }{ }^{\#}(n=270)\end{array}$ & $\begin{array}{l}10(1- \\
125)\end{array}$ & $12(1-125)$ & $8.5(1-72)$ & 0.126 \\
\hline $1-5$ sessions & $\begin{array}{l}93 \\
(34.4)\end{array}$ & $14(31.8)$ & $79(35)$ & \\
\hline 6-10 sessions & $\begin{array}{l}64 \\
(23.7)\end{array}$ & $4(9.1)$ & $60(26.5)$ & \\
\hline $11-15$ sessions & $35(13)$ & $9(20.5)$ & $26(11.5)$ & \\
\hline $16-20$ sessions & $\begin{array}{l}33 \\
(12.2)\end{array}$ & $9(20.5)$ & $24(10.6)$ & \\
\hline $21-25$ sessions & $16(5.9)$ & $3(6.8)$ & $13(5.8)$ & \\
\hline$>25$ sessions & 29 & $5(11.4)$ & $24(10.6)$ & \\
\hline
\end{tabular}


(10.7)

Number of treatment

sessions mentioned

Incomplete treatment

sessions mentioned $(n=42)$

Frequency of treatment sessions specified

Yes

No

NA

Frequency

of treatment (sessions/week, $n=246$ ) \#

1

3

$>3$

NA

Duration of treatment specified

Duration of treatment (weeks, $\mathrm{n}=\mathbf{2 6 0}$ )

$\bigotimes 4$

$4-8$

$9-12$

$>12$

NA

Patients posture specified

Disinfection specified
42

(13.2)

18

(42.9)
$29(10.4)$

0.005

0.021

0.317 *

$246 \quad 42(82.4) \quad 204(76.1)$

(77.1)

$23(7.2) \quad 1(2)$

$22(8.2)$

50

(15.7)

8 (15.7)

42 (15.7)

$3(1-14) \quad 3(1-14)$

$3(1-10)$

0.826

37 (15) $7(16.7)$

$30(14.7)$

54 (22) $9(21.4)$

$45(22.1)$

70

(28.5)

9 (21.4)

$61(29.9)$

$67 \quad 1(5.3)$

(27.2)

18 (7.3) 4 (9.5)

$14(6.9)$

260

$43(84.3)$

$217(81)$

0.573

(81.5)

$6(0-25 \rrbracket \quad 8(0-25)$

$4(0-24)$

0.003

73

(28.1)

141

(54.2)

$9(20.9)$

$64(29.5)$

$0.099 *$

$118(54.4)$

30

7 (16.3)

23 (10.6)

(11.5)

9 (3.5) $\quad 4$ (9.3)

$5(2.3)$

7 (2.7) $\quad 0(0)$

7 (3.2)

103

(32.3)

90

(28.2)
$16(31.4)$

87 (32.5)

0.879

$74(27.6)$

0.584 
Tble 3c: Other components of treatment and practitioner background

$\begin{array}{lllll}\text { Details of other interventions specified } & 73 & 17(33.3) & 56(20.9) & 0.053\end{array}$

Details of other interventions $\$$

TCM treatment (e.g., moxibustion, cupping)

$\begin{array}{llll}\text { Exercises } & 22 & 5(29.4) & 17(30.4)\end{array}$

Standard treatment or usual care 55 (75.3)

$6(8.2) \quad 1(5.9) \quad 5(8.9)$

Setting and context of treatment
specified

Details of participating acupuncturists specified

$\begin{array}{lll}232 & 34(66.7) & 198(73.9)\end{array}$

$41(73.2)$

Description of participating acupuncturists ${ }^{\wedge}$

$\begin{array}{llll}183 & 39(76.5) & 144(53.7) & 0.003 \\ (57.4) & \end{array}$

\begin{tabular}{|llll|}
$\begin{array}{c}\text { Qualification or professional } \\
\text { affiliation }\end{array}$ & $\begin{array}{l}112 \\
(61.2)\end{array}$ & $26(66.7)$ & $86(59.7)$ \\
\hline Years in acupuncture practice & $\begin{array}{l}110 \\
(60.1)\end{array}$ & $27(69.2)$ & $83(57.6)$ \\
\hline Other relevant experience & $\begin{array}{l}54 \\
(29.5)\end{array}$ & $16(41)$ & $38(26.4)$ \\
\hline
\end{tabular}

Abbreviations: NA, not applicable; TCM: traditional Chinese medicine. ${ }^{*}$ Fisher's exact test; others are Pearson's chi-squared test. \# Values are median (range) and $\mathrm{P}$ value are from Mann-Whitney $\mathrm{U}$ test. \& Fifty-one trials used two methods of needle manipulation, and eight trials used three methods of needle manipulation. \$ Fifty trials reported two details of electrical stimulation, and thirty-nine trials reported three details of electrical stimulation. $\$$ Ten trials reported two kinds of other interventions administered to the acupuncture group. ^Sixty-seven trials described two details of participating acupuncturists, and thirteen trials described three details of participating acupuncturists.

\section{Table 4 Selection and specification of control}




\begin{tabular}{|c|c|c|c|c|}
\hline Characteristics & $\begin{array}{l}\text { Total } \\
(\mathrm{N}=319, \\
\%)\end{array}$ & $\begin{array}{l}\text { Core Clinical } \\
\text { journals }(n=51, \%)\end{array}$ & $\begin{array}{l}\text { CAM journals } \\
(n=268, \%)\end{array}$ & $\begin{array}{l}P \\
\text { value }\end{array}$ \\
\hline Number of control group & & & & 0.62 \\
\hline 1 & $222(69.6)$ & $34(66.7)$ & $188(70.1)$ & \\
\hline$\geq 2$ & $97(30.4)$ & 17 (33.3) & $80(29.9)$ & \\
\hline $\begin{array}{l}\text { Both sham acupuncture and } \\
\text { waiting-list/blank control }\end{array}$ & $14(4.4)$ & $6(11.8)$ & $8(3.0)$ & \\
\hline Type of control ${ }^{*}$ & & & & $0.15^{\#}$ \\
\hline Sham acupuncture & $132(41.4)$ & $31(60.8)$ & $101(37.7)$ & \\
\hline Waiting-list/no treatment & $105(32.9)$ & $17(33.3)$ & $88(32.8)$ & \\
\hline Acupuncture & $66(20.7)$ & $9(17.7)$ & $57(21.3)$ & \\
\hline Standard care/usual care & $35(11.0)$ & $6(11.8)$ & $29(10.8)$ & \\
\hline $\begin{array}{l}\text { Other TCM treatment (e.g. } \\
\text { moxibustion, cupping) }\end{array}$ & $17(5.3)$ & $1(2.0)$ & $16(6.0)$ & \\
\hline Active medication & $47(14.7)$ & $3(5.9)$ & $44(16.4)$ & \\
\hline Surgery & $2(0.6)$ & $0(0)$ & $2(0.8)$ & \\
\hline Behavioral intervention & $17(5.3)$ & $4(7.8)$ & $13(4.9)$ & \\
\hline $\begin{array}{l}\text { Rationale for } \\
\text { control selection specified }\end{array}$ & $142(44.5)$ & $18(35.3)$ & $124(46.3)$ & 0.148 \\
\hline Precise description of the control & $282(88.4)$ & $48(94.1)$ & $234(87.3)$ & 0.164 \\
\hline
\end{tabular}

*97 trials included multiple control groups.

\#Fisher's exact test; others are Pearson's chi-squared test.

\section{Figures}




\section{Records identified from PubMed: $\mathbf{n}=\mathbf{1 8 5 0}$}

- Core clinical journals $(\mathrm{n}=339)$

- Complementary and alternative medicine journals $(n=1511)$

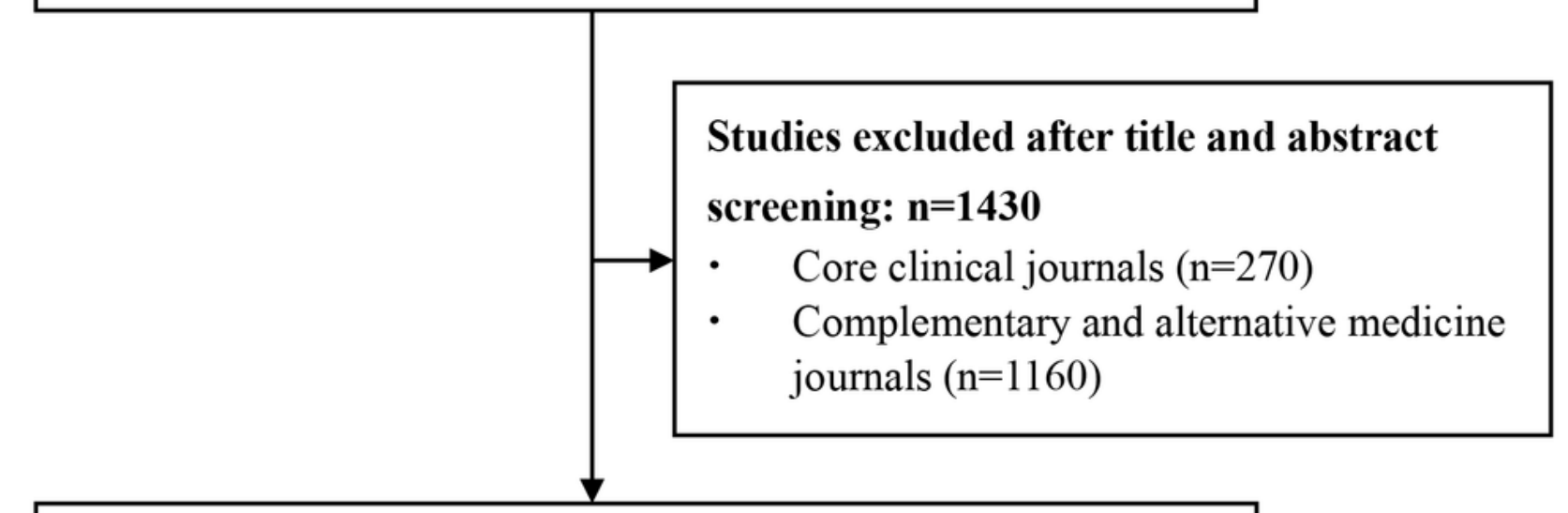

\section{Full-text screening: $n=420$}

- Core clinical journals $(n=69)$

- Complementary and alternative medicine journals $(n=351)$

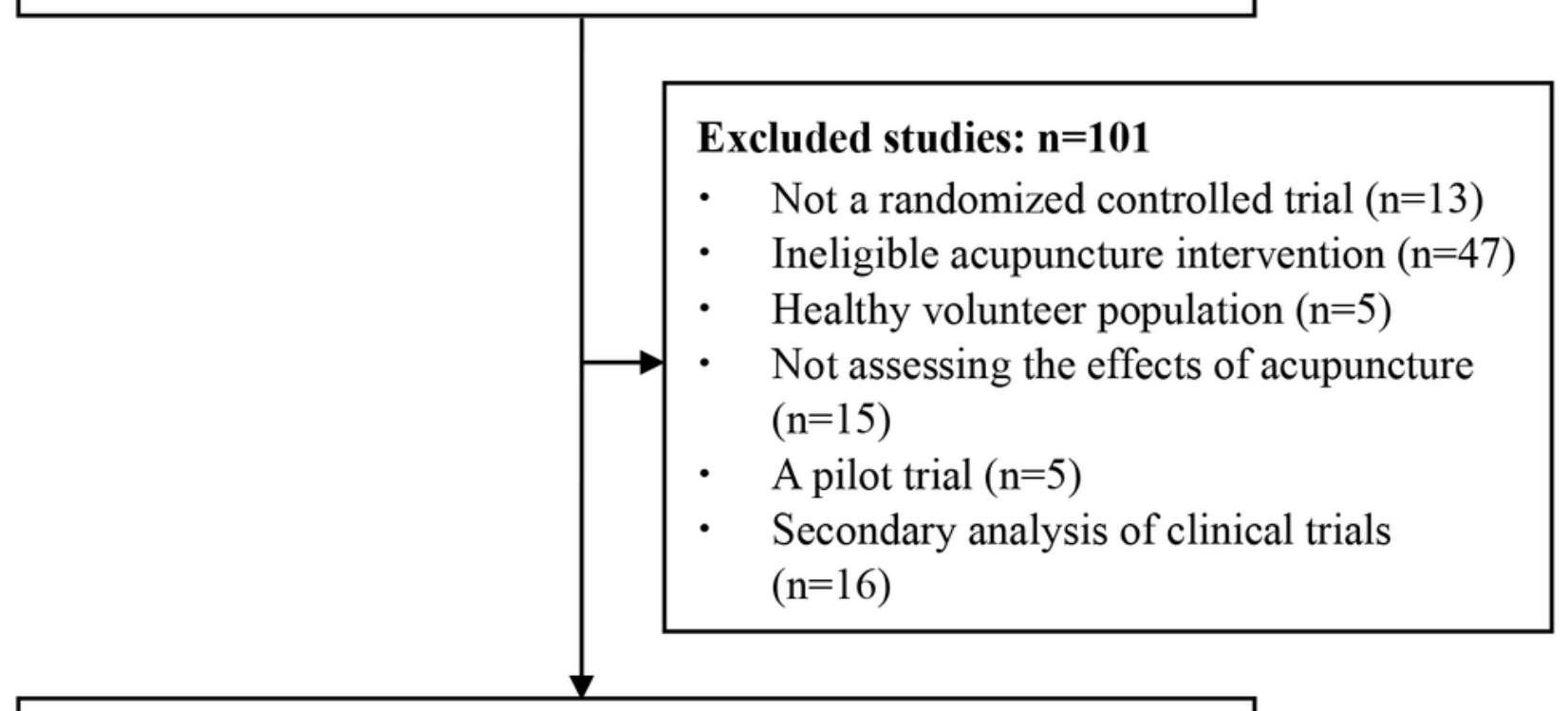

Trials included: $\mathbf{n}=\mathbf{3 1 9}$

- Core clinical journals $(\mathrm{n}=51)$

- Complementary and alternative medicine journals $(\mathrm{n}=268)$

\section{Figure 1}

Flow chart of study selection 


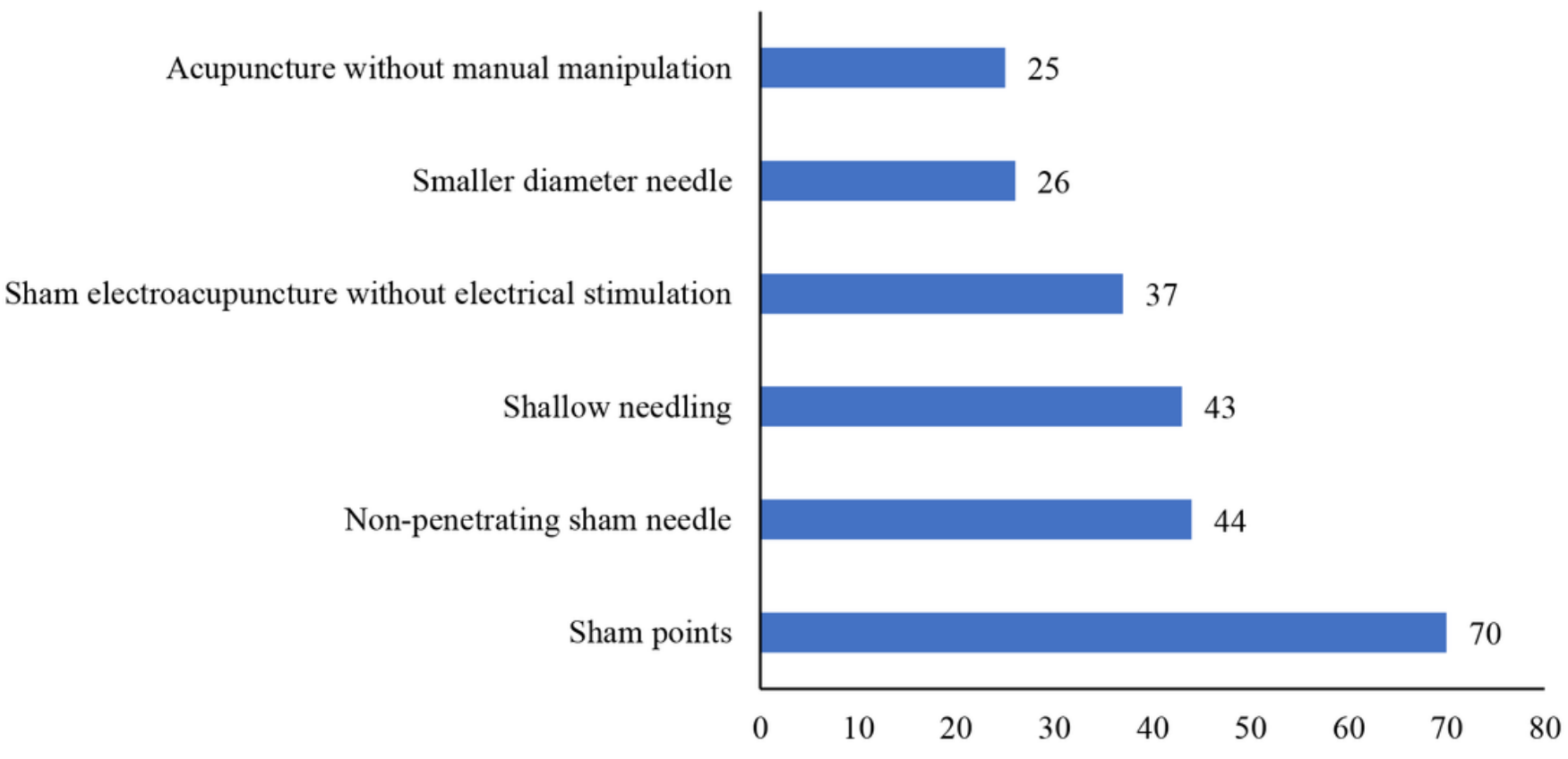

\section{Figure 2}

Sham acupuncture used in included trials

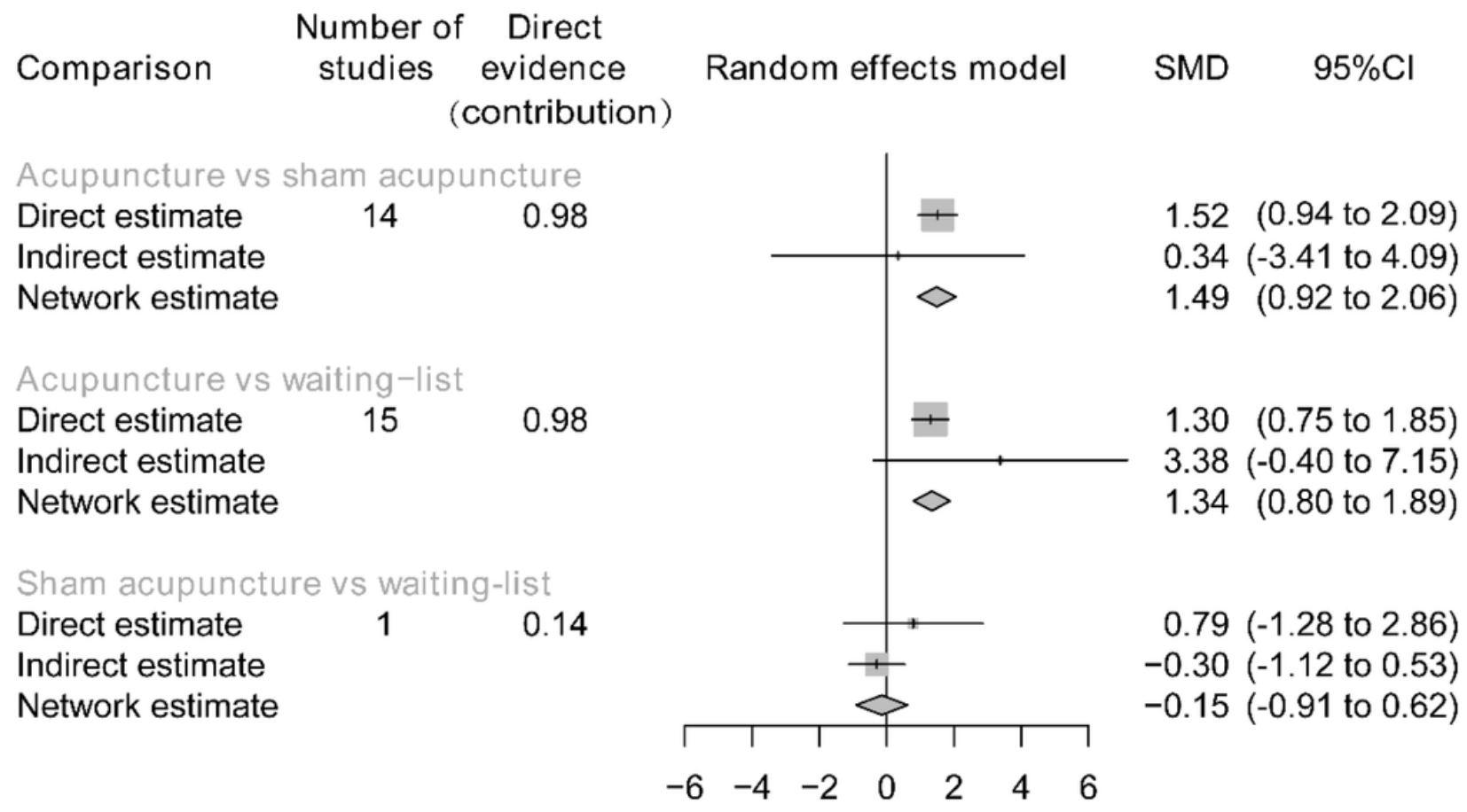

\section{Figure 3}


Network meta-analysis results of acupuncture, sham acupuncture and waiting-list/no treatment for chronic pain

\section{Supplementary Files}

This is a list of supplementary files associated with this preprint. Click to download.

- Appendix15.docx 